Originalveröffentlichung in: Friedrich, Julia ; Prinzing, Andreas (Hrsgg.): "So fing man einfach an, ohne viele Worte" :

Ausstellungswesen und Sammlungspolitik in den ersten Jahren nach dem Zweiten Weltkrieg, Berlin 2013, S. 18-25

\title{
Die theoretischen Diskurse über moderne Kunst in der Nachkriegszeit
}

\author{
Christoph Zuschlag
}

Die theoretischen Diskurse über moderne Kunst im Deutschland der Nachkriegszeit in einem Aufsatz angemessen zu würdigen, ist unmöglich. So muss ich mich auf Stichworte und Schlaglichter beschränken. Dennoch bin ich froh, dass das Thema hier zur Sprache kommt, weil es mir zum Verständnis des Ausstellungswesens und der Sammlungspolitik der Museen nach 1945 notwendig scheint.

Doch wie lassen sich die kunsttheoretischen Diskurse - die Debatten, Kontroversen, Erörterungen, Gespräche - überhaupt greifen, wie und wo kristallisieren sie sich heraus, und wie geht man methodisch bei ihrer Analyse vor? Sicher ist, dass sie im Kontext der politisch-gesellschaftlichen und ökonomisch-sozialen Systeme gesehen werden müssen, innerhalb deren sie sich herausbilden. Sicher ist auch, dass sie von den einzelnen Faktoren des Kunstsystems geprägt werden, also von dem Geflecht aus berufsständischen Vereinigungen und Künstlergruppen, Kunstkritik und Publizistik, Kunsthändlern und -sammlern, Ausstellungswesen und musealer Ankaufspolitik, und dass die Künstlerausbildung dabei ebenso eine Rolle spielt wie die öffentliche Kunstförderung. Kunsttheoretische Diskurse entstehen nicht im luftleeren Raum, weswegen im Folgenden auch viel die Rede von Künstlergruppen, Ausstellungen und Publikationen sein wird.

\section{Die vier Besatzungszonen und die Entwicklung in Ost und West}

Die Kulturpolitik und die kunsttheoretischen Diskurse entwickelten sich in den ersten Nachkriegsjahren in den vier Besatzungszonen unterschiedlich. Obwohl viele Städte bis weit in die 1950er Jahre zerstört waren und es der Bevölkerung an lebensnotwendigen Gütern mangelte, begann man sofort nach Kriegsende mit dem Wiederaufbau der Städte, der Infrastruktur und damit auch der kulturellen Institutionen. So wurde schon am 3. Juli 1945 in Berlin der Kulturbund zur demokratischen Erneuerung Deutschlands gegründet. Zum ersten Präsidenten wurde Johannes R. Becher gewählt (der spätere erste Minister für Kultur der DDR), zu einem der Vizepräsidenten der Maler Karl Hofer. 
$\mathrm{Zu}$ Beginn war der Kulturbund eine interzonale und überparteiliche Einrichtung, die zahlreiche Veranstaltungen, insbesondere Ausstellungen, organisierte. Nach Gründung der DDR diente er der SED zur Schaffung einer sozialistischen Kultur. Ebenfalls in Berlin eröffnete im August 1945 die Galerie Gerd Rosen als erste private Kunstgalerie nach dem Krieg. In Köln eröffneten Ende 1945 die Galerie »Der Spiegel « von Eva und Hein Stünke und die Moderne Galerie des Künstlers Erich Mueller-Kraus. ${ }^{1}$

In der Sowjetischen Besatzungszone (SBZ) entwickelte sich Dresden zu einem kulturellen Zentrum. Hier gründeten bereits im November 1945 Edmund Kesting, Hermann Glöckner, Helmut Schmidt-Kirstein und andere Künstler die Gruppe Der Ruf. Es war die erste Künstlergruppe in der SBZ nach dem Zweiten Weltkrieg. Die an der klassischen Moderne und sozialistischen Ideen orientierte Gruppe bestand bis 1948. Nach ihrer Auflösung schlossen sich einige Mitglieder der im März 1947 ebenfalls in Dresden gegründeten Gruppe Das Ufer an. ${ }^{2}$

\section{6 fand in Dresden die Allgemeine Deutsche Kunstausstellung} statt, an die sich ein Kunstkongress anschloss. ${ }^{3}$ Einer der Veranstalter war der erwähnte Kulturbund. Dieser Ausstellung kommt ein hoher Rang zu, war sie doch die erste und wegen des bald beginnenden Kalten Krieges gewissermaßen auch letzte gesamtdeutsche Kunstausstellung nach dem Krieg. Sie versammelte knapp 600 Werke der klassischen Moderne sowie Beispiele des aktuellen Kunstschaffens aus fast dem ganzen Land (die vorgesehenen Arbeiten aus der Britischen Besatzungszone konnten diese aus ungeklärten Gründen nicht verlassen). Damit sollte zum einen die während der NS-Herrschaft verfemte Moderne rehabilitiert und zum anderen ein Querschnitt durch die jüngste Kunstproduktion gezeigt werden. Die Schau wurde in Presse und Öffentlichkeit kontrovers diskutiert. Hans Grundig, Rektor der Staatlichen Kunsthochschule in Dresden und Mitglied der Jury, schrieb: »Die Probleme der Malerei, der bildenden Kunst überhaupt, ob realistisch oder abstrakt, ob zeitnahe und für unsere künftige Kunstgestaltung entwicklungsfähig, das sind die Fragen. Alles dies ist zu klären notwendig. Aber daß diese Ausstellung überhaupt zur Tatsache werden konnte [...], ist wohl das Besondere und Positive. Zum ersten Male seit 1933 eine beinahe vollständige Übersicht über das Schaffen Deutschlands und zum ersten Male eine Interzonenschau von großer Bedeutung, die klärend über die Grenzen hinweg den Weg zur Einheit Deutschlands weist. " ${ }^{4}$ Doch der Weg führte bekanntermaßen keineswegs zur Einheit Deutschlands, sondern im Gegenteil zur Spaltung.
$1 \mathrm{Vgl.1945-1985.} \mathrm{Kunst} \mathrm{in} \mathrm{der} \mathrm{Bundes-}$ republik Deutschland, hrsg. von der Nationalgalerie Staatliche Museen Preußischer Kulturbesitz, Ausst.-Kat. Nationalgalerie Berlin, Berlin 1985, S. 454 f. Daniela Wilmes, Wettbewerb um die Moderne. Zur Geschichte des Kunsthandels in Köln nach 1945, Berlin 2012, S. 107-115.

2 Vgl. Petra Jacoby, Kollektivierung der Phantasie? Künstlergruppen in der DDR zwischen Vereinnahmung und Erfindungsgabe, Bielefeld 2007.

3 Vgl. Kurt Winkler, "Allgemeine Deutsche Kunstausstellung «, in: Stationen der Moderne. Die bedeutenden Kunstausstellungen des 20. Jahrhunderts in Deutschland, Ausst.-Kat. Berlinische Galerie im Martin-Gropius-Bau Berlin 1988-89, Berlin 1988, S. 352-360. Kathleen Schröter, "Kunst zwischen den Systemen. Die Allgemeine Deutsche Kunstausstellung" 1946 in Dresden «, in: Nikola Doll u.a. (Hrsg.), Kunstgeschichte nach 1945. Kontinuität und Neubeginn in Deutschland, Köln u.a. 2006, S. 211-237. 
Zum Kunstkongress schreibt Grundig: »Abstrakte oder realistische Kunst, das war die Frage, aber noch ist der Fall nicht restlos geklärt. $\aleph^{5}$

Es waren die SED und die Sowjetische Militäradministration, die für die SBZ die Frage nach der zukünftigen Kunst »klärten «. Unter deren Einfluss wurde den Künstlern zunehmend das Beispiel der Sowjetunion mit ihrem Sozialistischen Realismus nahegelegt, während alles andere als "bürgerlich dekadente", "volksfremde" und "formalistische « Kunst abgelehnt wurde. Der einflussreiche sowjetische Kulturoffizier Major Alexander Dymschitz eröffnete am 24. November 1948 mit einem Artikel in der Täglichen Rundschau, dem Organ der Sowjetischen Militäradministration, eine Kampagne gegen den sogenannten Formalismus und die gesamte moderne Kunst. ${ }^{6}$ Der Formalismusvorwurf traf abstrakte Strömungen ebenso wie Anknüpfungen an den Expressionismus, wie man sie etwa im Werk Karl Hofers beobachten kann. Stattdessen forderte die SED eine realistische, "volksnahe " Kunst, die den Aufbau des Sozialismus bejahte und optimistisch unterstützte. Ministerpräsident Otto Grotewohl machte am 1. September 1951 unmissverständlich klar: »Literatur und bildende Kunst sind der Politik untergeordnet [...]. Die Idee in der Kunst muß der Marschrichtung des politischen Kampfes folgen.[...] Was sich in der Politik als richtig erweist, ist es auch unbedingt in der Kunst. ${ }^{7}$

Auch in den westlichen Besatzungszonen kam es bald nach Kriegsende zur Gründung von Künstlergruppen, deren Aktivitäten die kunsttheoretischen Diskurse der Zeit maßgeblich prägten. Diese Gruppen bestanden oft nur wenige Jahre, boten ihren Mitgliedern aber in der schwierigen Nachkriegszeit wichtigen Rückhalt. Erwähnt seien etwa die Westfälische Sezession 1945 in Hagen und die Rheinische Künstler-Gemeinschaft in Köln. ${ }^{8}$ In Düsseldorf wurde die Rheinische Sezession 1946 wiedergegründet (sie war 1938 verboten worden). "Eine der vitalsten und vielleicht auch folgenreichsten Künstlervereinigungen der Nachkriegszeit ${ }^{9}$ war der junge westen, gegründet 1948 in Recklinghausen von Thomas Grochowiak, Emil Schumacher und anderen Künstlern. Die Gruppe war abstrakt ausgerichtet und suchte den Anschluss an die internationale Avantgarde. 1962 löste sie sich auf, der Kunstpreis junger westen wird bis heute alle zwei Jahre verliehen. Ebenfalls ungegenständlich arbeitende, vorwiegend ältere Künstler (darunter. Willi Baumeister und Rupprecht Geiger) bildeten 1949 in München die Vereinigung ZEN 49. ${ }^{10}$ Initiator war der britische Konsul und spätere Kunstkritiker John Anthony Thwaites. ${ }^{11}$ Eine von der Gruppe 1956/57 durchgeführte
5 Zit. nach Winkler 1988 (wie Anm. 3), S. 359.

6 Vgl. zur Formalismuskampagne Eckhart Gillen, Das Kunstkombinat DDR. Zäsuren einer gescheiterten Kunstpolitik, hrsg. vom Museumspädagogischen Dienst Berlin, Köln 2005, S. 34-40.

7 Zit. nach ebd., S. 36.

8 Vgl. Hans M. Schmidt, mEine Gemeinschaft Einsamer, eine Verbundenheit Selbständigerc. Künstlervereinigungen der Nachkriegszeitu, in: Aus den Trümmern. Kunst und Kultur im Rheinland und in Westfalen 1945-1952. Neubeginn und Kontinuität, hrsg. von Klaus Honnef und

Hans M. Schmidt, Ausst.-Kat. Rheinisches Landesmuseum Bonn u.a., Köln und Bonn 1985, S. 423-431. Vgl. ferner Christoph Zuschlag, wKünstlergruppen in Deutschland:ZEN 49, Gruppe 53 «, in: ders., $m$ Informel - Ecole de Paris - Abstract Expressionism - Cobra. Die Sammlung Kraft Bretschneider in der Stiftung Kunst und Recht «, in: Donata Bretschneider (Hrsg.), Tendenzen der abstrakten Kunst nach 1945. Die Sammlung Kraft Bretschneider in der Stiftung Kurist und Recht, Tübingen, Heidelberg 2003, S. 9-35, hier S. 22-25.

9 Schmidt 1985 (wie Anm. 8), S. 425.

$10 \mathrm{Vgl}$. ZEN 49. Die ersten zehn JahreOrientierungen, hrsg. von Jochen Poetter, Ausst.-Kat. Baden-Baden 1986-87, Stuttgart-Bad Cannstatt [1986]; Beate Frosch, Die Künstlergruppe ZEN 49 und ihr Beitrag zur Entwicklung der gegenstandslosen Kunst in der Bundesrepublik Deutschland 1949-1957, Regensburg 1992; Iris Buchheim und Cathrin Klingsöhr-Leroy, ZEN 49. Fragmente der Erinnerung, Ausst-Kat. München 1999-2000, Ostfildern [1999].

11 Vgl. Christoph Zuschlag, mVive la critique engagée!c. Kunstkritiker der Stunde Null: John Anthony Thwaites (1909-1981) «, in: Brennpunkt Informel. Quellen - Strömungen - Reaktionen, hrsg. von Christoph Zuschlag u. a., Ausst. Kat. Kurpfälzisches Museum der Stadt Heidelberg und Heidelberger Kunstverein 1998-99, Köln 1998, S. 166-172. Beate Eickhoff, John Anthony Thwaites und die Kunstkritik der 50er Jahre, Weimar 2004. 
Ausstellungstournee durch die USA markierte den Höhepunkt und zugleich in etwa das Ende von ZEN 49.

\section{Figuration versus Abstraktion}

Der zentrale kunstkritische und -theoretische Diskurs kreiste ohne Zweifel um das Thema gegenständlich-figurative versus abstrakt-ungegenständliche Kunst. Eine der frühesten Manifestationen der abstrakten Kunst nach 1945 ereignete sich im Rheinland. Auf Schloss Alfter bei Bonn bildete sich 1947 die Donnerstag-Gesellschaft, ein loser Zusammenschluss von Malern, Schriftstellern und Musikern, die bis $1950 \mathrm{zu}$ Lesungen, Vorträgen, Diskussionen, Konzerten und Ausstellungen einluden. ${ }^{12}$ Am 20. Juli 1947 veranstaltete sie einen Tag der Abstrakten Kunst wohl die allererste Ausstellung abstrakter Kunst nach Ende des Zweiten Weltkrieges in Deutschland. Sie umfasste 32 Arbeiten von Eugen Batz, Hubert Berke, Joseph Fassbender, Georg Meistermann, Erich Mueller-Kraus und Hann Trier. Die Begrüßung übernahm Josef Haubrich, den Eröffnungsvortrag Werner Haftmann. Als um die Jahreswende 1948/49 die von dem Stuttgarter Sammler Ottomar Domnick organisierte Wanderausstellung französischer abstrakter Malerei in Düsseldorf Station macht, schreibt Anna Klapheck in der Westdeutschen Rundschau: »Der Durchbruch der abstrakten Kunst in allen Ländern muß als Tatsache hingenommen werden. « ${ }^{13}$

Ich hatte eingangs die Frage gestellt, wie sich die kunsttheoretischen Diskurse überhaupt greifen lassen. Ein für diese Epoche signifikantes Format war das öffentliche Expertengespräch, das häufig in Verbindung mit einer Ausstellung organisiert wurde und durch Funk und Fernsehen eine breite Öffentlichkeit erreichte. Die Verschiebung der Diskurse in den fünfziger Jahren lässt sich wie in einem Nukleus an drei Gesprächen in Darmstadt (1950), Leverkusen (1956) und Baden-Baden (1959) nachvollziehen.

\section{Das Darmstädter Gespräch 1950}

1950 fand in Darmstadt auf der Mathildenhöhe die von der Neuen Darmstädter Sezession organisierte Ausstellung das menschenbild in unserer zeit statt. Im Katalog schreibt Adolf Schmoll gen. Eisenwerth: "Im Streit um das Menschenbild der Gegenwart stehen sich [...] nicht nur die Vertreter verschiedener persönlicher Meinungen gegenüber, sondern diejenigen verschiedener Epochen, um nicht pathetisch zu sagen: verschiedener Welten. [...] Das einzig Sichere bleibt die Erkenntnis von einer umstürzen-
12 Vgl. Die Donnerstag-Gesellschaft Alfter 1947-1950. Eine Dokumentation von Frank-R. Hildebrandt und Jens Scholz, Düsseldorf 1997; Hans M. Schmidt, "Die Donnerstag-Gesellschaft zu Alfter. Eine ungewöhnliche Kultur-Initiative der Nachkriegszeit«, in: Dieter Breuer und Gertrude Cepl-Kaufmann (Hrsg.), Öffentlichkeit der Moderne - Die Moderne in der Öffentlichkeit. Das Rheinland 1945-1955. Vorträge des interdisziplinären Arbeits kreises zur Erforschung der Moderne im Rheinland, Essen 2000, S. 141-156. Vgl. auch Wilmes 2012 (wie Anm. 1), S. 149-158.

13 Westdeutsche Rundschau, 15. Januar 1949. Zit. nach Gabriele Lueg, Im Spiegel der Presse. Die Kunstkritik in der Nachkriegszeit, in: Honnef und Schmidt 1985 (wie Anm. 8), S. 443-449, hier S. 444. 
den Wandlung. Das Menschenbild ist in ihren Strudel gerissen worden. Am jenseitigen Ufer erhebt sich die sabsoluter, sungegenständliche Kunst - für die einen die Erscheinung des schrecklich Sinnleeren oder gar Sinnlosen, für die anderen das SchöpferischBefreiende $[\ldots] . \ll^{14}$

Im Rahmen der Ausstellung fand vom 15. bis 17. Juli 1950 eine Gesprächsrunde statt, die als Erstes Darmstädter Gespräch in die Geschichte eingehen sollte. ${ }^{15}$ An ihm nahmen führende Künstler und Kunstkritiker, aber auch Wissenschaftler anderer Disziplinen teil. Exemplarisch seien genannt der Philosoph und Soziologe Theodor W. Adorno und der Psychoanalytiker Alexander Mitscherlich. Hauptkontrahenten waren der Kunsthistoriker Hans Sedlmayr und Willi Baumeister. Sedlmayrs in den 1930er Jahren begonnene Studie Verlust der Mitte mit dem Untertitel Die bildende Kunst des 19. und 20. Jahrhunderts als Symptom und Symbol der Zeit erschien 1948 in der ersten und 1951 bereits in vierter Auflage, der rasch weitere folgen sollten. Das Buch fand auch als Taschenbuch weite Verbreitung und wurde in mehrere Sprachen übersetzt. Von einem konservativ-kulturkritischen, religiös geprägten Standpunkt aus beschreibt Sedlmayr mit medizinischem Vokabular die Entwicklung der Kunst vom späten 18. Jahrhundert bis zur Moderne als die eines allmählichen Verfalls, die ihre Ursache in einer zunehmenden Entfernung von einem geordneten, christlich bestimmten Weltbild mit festen Leitbildern und Werten habe. In der Abstraktion und dem Verlust des Menschenbildes habe dies schließlich zum »Verlust der Mitte " geführt und folglich zu einer exzentrischen, chaotischen Kunst: »Die Kunst strebt fort vom Menschen, vom Menschlichen und vom Maß. ${ }^{16}$

Sedlmayrs Antipode auf der Seite der Befürworter der Abstraktion war Willi Baumeister, Professor an der Stuttgarter Kunstakademie und ZEN 49-Gründungsmitglied, dessen ebenfalls in den Kriegsjahren verfasstes Buch Das Unbekannte in der Kunst 1947 erschienen war. Baumeister sah die Entwicklung der bildenden Kunst »als eine fortschreitende Befreiung von Religion, Auftrag und Inhalt. Für Baumeister stellte die abstrakte Kunst »die höchste Stufe der bisherigen Kunstentwicklung dar [... « «. ${ }^{17}$ Der Künstler plädierte für eine ethisch motivierte, gegenstandsfreie Kunst, die zum Unbekannten vorstößt: »Der originale Künstler verläßt das Bekannte und das Können. Er stößt bis zum Nullpunkt vor. Hier beginnt sein hoher Zustand $«{ }^{18}$ Im Darmstädter Gespräch kulminierte die zentrale kunsttheoretische Debatte der unmittelbaren Nachkriegszeit. Der Hessische Rundfunk schnitt das Gespräch mit und sendete an zwei Terminen Ausschnitte daraus.
14 Adolf Schmoll gen. Eisenwerth, "Zur Ausstellung 'Das Menschenbild in unserer Zeitu, in: das menschenbild in unserer zeit, Ausst.-Kat. Mathildenhöhe Darmstadt, Darmstadt [1950], o. S.

15 Vgl. Hans Gerhard Evers (Hrsg.) Das Menschenbild in unserer Zeit, Darmstadt [1950].

16 Hans Sedlmayr, Verlust der Mitte. Die bildende Kunst des 19. und 20. Jahrhunderts als Symptom und Symbol der Zeit, ungekürzte Ausgabe nach der 10. Auflage, Frankfurt am Main und Berlin 1988, S. 148

17 Philipp Gutbrod, "Werner Haftmanns Einführung im Katalog der »documenta 2 «", in: Michael Glasmeier und Karin Stengel (Hrsg.), archive in motion. documentaHandbuch. documenta manual, Ausst.-Kat. Kunsthalle Fridericianum Kassel, Göttingen 2005, S. 191-200, hier S. 192. Vgl. auch ders., "Baumeister versus Sedlmayr. Die Kontroverse um Kunst und Religion im ersten Darmstädter Gespräch (1950)«, in: Kirsten Fitzke und Zita Ágota Pataki (Hrsg.), Kritische Wege zur Moderne. Festschrift für Dietrich Schubert, Stuttgart 2006, S. 43-67.

18 Willi Baumeister, Das Unbekannte in der Kunst, Stuttgart 1947, S. 155. 


\section{Das Leverkusener Gespräch 1956}

Ein anderes Gespräch geriet unverdientermaßen fast in Vergessenheit: das Leverkusener Gespräch. ${ }^{19}$ Auch dieses stand in Verbindung mit einer Ausstellung. Vom 28. November 1956 bis 2. Januar 1957 fand im Museum Morsbroich der Stadt Leverkusen die Ausstellung Malerei und Plastik in Westdeutschland 1956 statt. Das Unternehmen war hochambitioniert, ging es doch um eine Bestandsaufnahme der aktuellen Kunst in der Bundesrepublik. Veranstalter war der Westdeutsche Künstlerbund. Dessen Vorsitzender Wilhelm Wessel schrieb in seinem Katalogvorwort, die Ausstellung wolle »Tendenzen der westdeutschen Kunst von heute sichtbar « machen, wobei eine "jüngere Schicht " von Künstlern im Vordergrund stehe. ${ }^{20}$ Ungegenständlich arbeitende Künstler dominierten, wenngleich mit Erich Heckel auch ein Repräsentant des Brücke-Expressionismus vertreten war. Von Anfang an hatte Wessel geplant, ausländische Experten um eine schriftliche Begutachtung der Ausstellung zu bitten und diese zusammen mit Pressekritiken zu veröffentlichen. Die Stellungnahmen sowie die aus- und inländische Presse wurden 1957 in einer eigenen Broschüre dokumentiert. ${ }^{21}$

Bereits drei Tage nach Eröffnung trafen auf Einladung Wilhelm Wessels im Museum renommierte Kunstexperten aus mehreren europäischen Ländern zusammen: die Kunsthistoriker und -kritiker Ernest Goldschmidt (Brüssel), Will Grohmann (Berlin), Giuseppe Marchiori (Venedig), Herbert Read (London), Michel Tapié (Paris) und Herta Wescher (Paris), sodann die Museumsleiter Pierre Janlet (Brüssel), Franz Meyer (Bern), Willem J. H. B. Sandberg (Amsterdam) und Georg Schmidt (Basel) sowie der Galerist Rodolphe Stadler (Paris).

Nach einem Rundgang diskutierten die Experten die Fragen, ob der Durchbruch der ungegenständlichen Kunst nach 1945 in den verschiedenen Ländern als Ausdruck der Befreiung zu verstehen sei, ob innerhalb der internationalen Bewegung in den verschiedenen Ländern nationale Wesenszüge zu erkennen seien und ob die deutsche Kunst die durch die NS-Zeit bedingte Phase der Isolierung überwunden habe. Sie waren überwiegend der Meinung, dass die (ungegenständliche) Kunst der Gegenwart insgesamt eher international denn national oder regional sei und dass Deutschland elf Jahre nach Kriegsende wieder den Anschluss an die internationale Avantgarde gefunden habe. Die Frage, ob der Durchbruch der Abstraktion nach 1945 Ausdruck der »libération« sei, beantwortete zum Beispiel Georg Schmidt "für Deutschland und Italien mit einem klaren Ja«. Zugleich warnte er davor, »den Geist
$19 \mathrm{Vgl.}$ 1945-1985. Kunst in der Bundesrepublik Deutschland (wie Anm. 1), S. 499; Christoph Zuschlag, "Zur Debatte um ungegenständliche Kunst in den $50 \mathrm{er}$ Jahren: Das , Leverkusener Gespräch««, in: Doll u.a. 2006 (wie Anm. 3), S. 183-194.

20 Wilhelm Wessel, in: Malerei und Plastik in Westdeutschland 1956, Ausst.-Kat. Museum Morsbroich der Stadt Leverkusen, Bonn [1956], o. S.

21 Dokumentation Westdeutscher Künstlerbund - Die Meinungen ausländischer Experten und die Meinungen deutscher Kunstkritiker - zur Ausstellung 'Malerei und Plastik in Westdeutschland 1956, Leverkusen, Hagen 1956. 
der >libération` mit dem Geist der ungegenständlichen Kunst zu identifizieren und die slibération' gar als Vater der ungegenständlichen Kunst zu bezeichnen. Die >libération« war vielmehr ein Sammelbecken sämtlicher von den Diktaturen verfolgten geistigen Bewegungen." Auch Ernest Goldschmidt bestritt, dass die Abstraktion eine Folge der Befreiung gewesen sei, vielmehr habe diese lediglich neuen Schwung in eine bereits seit Anfang des Jahrhunderts sich entwickelnde Richtung gebracht. ${ }^{22}$ AnschlieBend fuhren die Kritiker nach Köln, wo im WDR ein Rundfunkgespräch über diese Fragen stattfand, das mit einer Einführung und einem Schlusswort von Carl Linfert am Abend des 1. März 1957 gesendet wurde. $^{23}$

\section{Das Baden-Badener Gespräch 1959}

Unter der Fragestellung: »Wird die moderne Kunst sgemanagtı? « veranstaltete der Verlag der Zeitschrift Das Kunstwerk Ende Oktober 1959 - die II. documenta hatte gerade ihre Pforten geschlossen - in der Staatlichen Kunsthalle Baden-Baden das erste von mehreren geplanten öffentlichen Baden-Badener Kunstgesprächen, das zum Teil als Fernsehgespräch mit Publikumsbeteiligung stattfand und nach Aussage der Veranstalter auf »ein überraschendes Echo im In- und Ausland ${ }^{24}$ stieß. Teilnehmer waren unter anderem der Philosoph Max Bense, der Soziologe Arnold Gehlen, der Künstler HAP Grieshaber, der Kunsthändler DanielHenry Kahnweiler, der Sammler Karl Ströher, der Kunstkritiker Egon Vietta und der Kunsthistoriker Leopold Zahn. Die Gegner der abstrakten Kunst wie Hans Sedlmayr hatten die Einladung abgelehnt, der Beitrag von Theodor W. Adorno wurde in dessen Abwesenheit verlesen. Anlass der Debatte war die in den Medien geäußerte Kritik an der Jury des in Baden-Baden vergebenen Ersten Deutschen Kunstpreises der Jugend. Der Vorwurf lautete, die Künstlermitglieder stünden überwiegend dem Tachismus nahe und der künstlerische Nachwuchs solle, so Egon Vietta in der Zeitschrift Das Kunstwerk, »in dirigistischer Weise im Sinne einer sehr einseitig orientierten künstlerischen Ausdrucksform beeinflußt werden $«{ }^{25}$ Ein weiterer Auslöser war zweifellos die II. documenta. An ihr wurde die Bevorzugung ungegenständlicher Richtungen ebenso kritisiert wie die Einflussnahme durch Kunsthändler, die als wichtige Leihgeber fungierten. Den im Raum stehenden Vorwurf der Manipulation durch Kunstmarkt und Kunstbetrieb brachte ein Teilnehmer in Baden-Baden mit den Worten auf den Punkt: »Die Nichtgegenständlichen werden gemanagt, die Gegenständlichen nicht - siehe documenta II da sitzt das Unbehagen! ${ }^{26}$
22 Zitatnachweise bei Zuschlag 2006 (wie Anm. 19), S. 189.

23 Eine Aufzeichnung befindet sich im Archiv des WDR in Köln.

24 Vorbemerkung des Verlags, in: Wird die moderne Kunst gemanagt? Baden-Baden und Krefeld 1959, S. 7. Vgl. jüngst Hendrik Bündge, "Die Documenta als Katalysator für Kunst und Gesellschaft", in: Bilderbedarf. Braucht Gesellschaft Kunst? The Civic and the Arts, hrsg. von Johan Holten, Ausst.-Kat. Staatliche Kunsthalle BadenBaden, Köln 2012, S. 35-49, hier S. 45 f. Hingewiesen sei auf die titellose Zeichnung von Marcel van Eeden aus dem Jahr 2009, die den Schriftzug "WIRD DIE MODERNE KUNST , GEMANAGT? « unter einer von einer Gabel aufgespießten Wurst zeigt. Vgl. die Abb. in: Michael Zink, Marcel van Eeden. Wird die moderne Kunst "gemanagt«? Zeichnungen und Malerei 1992-2009, Köln [2009], S. 314.

25 Zit. nach 1945-1985. Kunst in der Bundesrepublik Deutschland (wie Anm. 1) S. 510. Preisträger war Friedrich Werthmann. Weitere Auszeichnungen erhielten Horst Antes, Emil Cimiotti, Otto Piene und Heimrad Prem (vgl. ebd.).

26 Wird die moderne Kunst gemanagt? (wie Anm. 24), S. 93. 


\section{Fazit und Ausblick}

Auch wenn ich hier nur einen sehr kursorischen Blick auf das Thema werfen konnte, so zeigt sich doch Folgendes: Der zentrale kunsttheoretische Diskurs der Zeit kreiste um die Frage Figuration oder Abstraktion. In der SBZ bestimmten die Sowjetische Militäradministration und die SED den Diskurs - "Formalismuskampagne « und »Sozialistischer Realismus « lauten die Stichworte. Im Westen kulminierte die Auseinandersetzung um gegenständliche und ungegenständliche Kunst und um das Menschenbild in der Moderne zum ersten Mal im Darmstädter Gespräch 1950. Beim Leverkusener Gespräch Ende 1956, ein Jahr nach der ersten documenta, war die historische und kunsthistorische Situation schon eine gänzlich andere. In den Pariser Verträgen vom 5. Mai 1955 hatten die Westmächte die BRD in die Unabhängigkeit entlassen und ihr die Aufstellung eigener Streitkräfte im Rahmen der Westeuropäischen Union und der NATO ermöglicht. Bundeskanzler Konrad Adenauer setzte seine Politik der Westintegration fort. Außenpolitisch wuchs die Rolle der BRD in der internationalen Staatengemeinschaft, während die Wirtschaft im Lande prosperierte ("Wirtschaftswunder"). Es waren die Jahre des Kalten Krieges, in dem auch die Kunst instrumentalisiert wurde (und zwar in beiden deutschen Staaten). In Leverkusen wurde die mittlerweile dominierende Rolle der ungegenständlichen Kunst nicht mehr bezweifelt. Ziel war es zu demonstrieren, dass die deutsche Kunst wieder Anschluss an die internationale Avantgarde gefunden hatte, und dies sollte durch die Sicht von außen bestätigt werden. Während es in Darmstadt in heftigen Diskussionen um die Alternative gegenständliche oder ungegenständliche Kunst ging und man darin regelrecht zwei gegensätzliche Ideologien sah, herrschte in Leverkusen weitgehend Harmonie. Hier hatten sich Befürworter und Förderer nichtfigurativer Kunst zusammengefunden, deren Anschauungen in einzelnen Punkten differierten, nicht aber grundsätzlich voneinander abwichen. 1959, im Jahr des Baden-Badener Gesprächs, standen erneut andere Fragen im Mittelpunkt der Diskurse. Im selben Jahr hatte Werner Haftmann für die II. documenta die nicht unumstrittene Devise $"$ Die Kunst ist abstrakt geworden ${ }^{27}$ ausgegeben. Die junge deutsche Kunst war in Kassel ganz selbstverständlich als Teil der internationalen Avantgarden gezeigt worden. Vom Gefühl der Isolation also keine Spur mehr. In Baden-Baden rückte nun erstmals der ökonomische Aspekt, in den Mittelpunkt. Die Kritiker der Abstraktion bzw. deren vermeintlich einseitiger Bevorzugung warfen Handel und Kunstbetrieb Dirigismus und Manipulation vor. Zu diesem Zeitpunkt hatte sich die künstlerische Avantgarde längst in andere Richtungen bewegt - „Neue Figurationen" hatten sich manifestiert, die Gruppen Zero und Spur sich gebildet, und nur wenig später sollte die Fluxus-Bewegung Kunstbegriff und Kunstbetrieb gehörig aufmischen. 